\title{
REM sleep behavior disorder, neurodegeneration and Wilson's disease
}

Distúrbio comportamental do sono REM, neurodegeneração e doença de Wilson

João Carlos Papaterra Limongi

Departamento de Neurologia da Faculdade de Medicina da Universidade de São Paulo, São Paulo SP, Brazil.

\section{Correspondence:}

João Carlos Papaterra Limongi;

Rua Dr Alceu de Campos

Rodrigues, 46 / conj. 103;

04544-000 São Paulo SP, Brasil;

E-mail: limongi@uol.com.br

Conflict of interest:

There is no conflict of interest to declare.

Received 02 August 2014 Accepted 22 August 2014

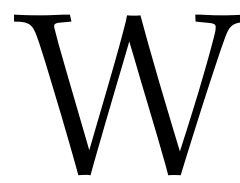

ilson's disease (WD) is an autosomal recessive inherited disorder of copper metabolism resulting in pathological accumulation of copper in the brain among other organs. The disease is associated with deficiency of a coppertransporting ATPase resulting from one of several possible mutations in the ATP7B gene located on chromosome 13 (q14.3) ${ }^{1}$. Mutations lead to reduced copper binding by ceruloplasmin with free copper accumulating mainly in the liver, cornea and different parts of the brain predominantly in basal ganglia. Putamen and globus pallidus are particularly affected but pathological changes are also observed in the caudate nucleus, internal capsule, substantia nigra, thalamus, cerebral cortex, subcortical white matter, subthalamic nucleus, cerebellum and brainstem.

Symptoms of copper accumulations range from neurological and psychiatric disturbances to acute or chronic liver disease. The neurological symptoms, present in about $40 \%$ of affected patients, include dysarthria, dyspraxia, ataxia and parkinsonism. A variety of sleep disturbances including disorders of rapid eye movements (REM) sleep have been described ${ }^{2,3}$.

Neuroimaging findings in WD suggest a complex pattern of nigrostriatal dopaminergic disruption and reduced glucose metabolism in several brain regions. These observations, together with impairment of the descending frontobulbar saccadic pathway, cortico- motoneural pathway, somatosensory, auditory and visual pathways at the brainstem level revealed by neurophysiological studies might explain some of sleep disorders seen in $\mathrm{WD}^{4,5}$.

REM sleep behavior disorder (RBD) is a parasomnia that is characterized by elaborate and often violent motor behaviors during REM sleep ${ }^{6}$. During normal REM sleep, movements are absent because skeletal muscles are effectively paralyzed. However, in patients with RBD, this phenomenon is impaired and a variety of motor behaviors can occur. Behaviors range from simple motor activities, such as talking, shouting, and limb jerking, to more complex movements that are seemingly purposeful and goal directed, such as gesturing, punching, or kicking. Motor behaviors in RBD can result in injury to the patient or to the spouse that often requires medical attention. RBD is a major concern because most patients eventually develop a neurodegenerative disease generally associated with alfa-synuclein deposition. More than $80 \%$ of patients with RBD eventually develop Parkinson's disease (PD), multiple system atrophy (MSA), or dementia with Lewy bodies (DLB). RBD is considered a reliable clinical predictor of onset of neurodegenerative diseases and the ability to identify prodromal neurodegeneration before actual clinical onset has major implications. RBD presents a unique opportunity to study the development of a neurodegenerative syndrome from its prodromal stages. In clinical trials, the study of RBD may be the ideal way to develop neuroprotective therapies for prevention of PD, MSA, and DLB since treatment can start early enough to effective intervention and before symptomatic PD medications confound assessments.

In spite of the well-recognized relationships between WD and PD and the presence of a wide spectrum of sleep disorders in WD, the association between WD and RBD has not been fully appreciated. In the article by Tribl GG et al., in this issue, four cases of early neurological WD patients presenting with RBD are described ${ }^{7}$. All cases underwent extensive structured clinical and sleep interviews with the patients and their caregivers and were submitted to specific questionnaires for RBD, cognitive evaluation, two-week dream diary, polissomnography and transcranial 
sonography (TCS). In three cases, RBD was the initial manifestation of WD. All four cases show TCS hyperintensities of midbrain tegmentum which is the location considered crucial for the genesis of idiopathic RBD and of RBD in PD.

In alfa-synucleinopathies, RBD is a highly frequent early stage manifestation of neurodegeneration ${ }^{8}$. Although considered a disease of metabolism, WD shares with PD not only the clinical phenomenology but also a similar topographical distribution of basal nuclei and brainstem lesions and the accumulation of metals in these locations. As the authors point out, the tegmental lesions in MRI and TCS do not prove a necessary causal relationship between WD and RBD, as it is already established in PD and RBD. But the presented cases could provide some evidence for both RBD as a clinical symptom in early brain damage in $\mathrm{WD}$, and for tegmental lesions as a possible causal factor for RBD. It is also possible that, in special cases, early diagnosis could allow for prompt initiation of copper eliminating therapy thus contributing to minimize brain damage and to provide more favorable results with long term pharmacological therapy.

\section{References}

1. Tanzi RE, Petrukhin K, Chernov I, et al. The Wilson disease gene is a copper transporting ATPase with homology to the Menkes disease gene. Nat Genet 1993;5:344-350.

2. Nevsimalova S, Buskova J, Bruha R, et al. Sleep disorders in Wilson's disease. Eur J Neurol 2011;18:184-190.

3. Firneisz G, Szalay F, Halasz P. Hypersomnia in Wilson's disease: an usual symptom in an unusual case. Acta Neurol Scand 2000;101:286-288.

4. Westermark K, Tedroff J, Thuomas KA, et al. Neurological Wilson's disease studied with magnetic resonance imaging and with positron emission tomography using dopaminergic markers. Mov Disord 1995;10:596-603.
5. Hawkins RA, Mazziotta JC, Phelps ME. Wilson's disease studied with FDG and positron emission tomography. Neurology 1987;37:1707-1711.

6. Peever J, Luppi PH, Montplaisir J. Breakdown in REM sleep circuitry underlies REM sleep behavior disorder. Trends Neurosci 2014;37:279-288.

7. Tribl GG, Shu EBS, Trindade MC, et al. Wilson's disease presenting as rapid eye movement sleep behavior disorder: a possible window to earl treatment. Arq Neuropsiquiatr 2014;72:xx-Xx.

8. Postuma RB, Gagnon JF, Montplaisir JY. REM sleep behavior disorder: from dreams to neurodegeneration. Neurobiol Dis 2012;46:553-558. 